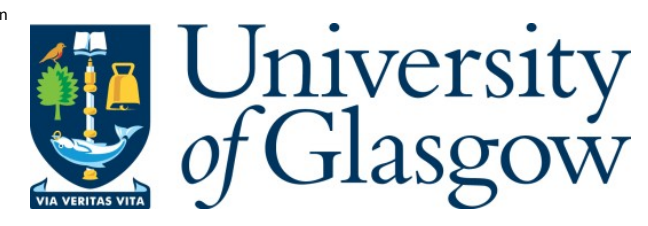

B arr, M ., Fahy, A ., Jardine, A ., Ellis, J., W ard, D., M acL aren, D.A., Allison, W., and Dastoor, P.C. (2014) A design for a pinhole scanning helium microscope. Nuclear Instruments and M ethods in Physics Research Section B: B eam Interactions with M aterials and A toms, 340 . pp. 76-80. ISSN 0168-583X

Copyright @ 2014 Elsevier B.V.

A copy can be downloaded for personal non-commercial research or study, without prior permission or charge

Content must not be changed in any way or reproduced in any format or medium without the formal permission of the copyright holder(s)

http://eprints.gla.ac.uk/100654/

Deposited on: 18 December 2014

Enlighten - Research publications by members of the U niversity of Glasgow http://eprints.gla.ac.uk 


\title{
A design for a pinhole scanning helium microscope
}

\author{
M Barr1', A Fahy'1, A Jardine², J Ellis², D. Ward2, D A MacLaren³, W Allison'2, and P C Dastoor1 \\ ${ }^{1}$ Centre for Organic Electronics, University of Newcastle, Callaghan, NSW 2308, Australia. \\ ${ }^{2}$ Cavendish Laboratory, University of Cambridge, Madingley Road, Cambridge, CB3 0HE \\ ${ }^{3}$ Dept. of Physics, University of Glasgow, Glasgow G12 8QQ, UK \\ E-mail: Paul.Dastoor@newcastle.edu.au
}

Keywords: helium atom scattering, helium atom microscopy, scanning helium microscope, SHeM

\begin{abstract}
We present a simplified design for a scanning helium microscope (SHeM) which utilises almost entirely off the shelf components. The SHeM produces images by detecting scattered neutral helium atoms from a surface, forming an entirely surface sensitive and non-destructive imaging technique. This particular prototype instrument avoids the complexities of existing neutral atom optics by replacing them with an aperture in the form of an ion beam milled pinhole, resulting in a resolution of around 5 microns. Using the images so far produced, an initial investigation of topological contrast has been performed.
\end{abstract}

\section{Introduction}

For the particles and photons typically used in microscopy studies, a reduction in wavelength leads to an increase in resolution with a corresponding increase in probe energy. Since bond energies are much lower than the kinetic energy of the impinging probe particles, delicate samples (such as organic thin films and adsorbate structures) can be degraded during the imaging process [1-3]. If neutral helium atoms are used instead of electrons or photons, short wavelengths $(\sim 0.5 \AA)$ are achieved at low kinetic energies $(\sim 20 \mathrm{meV})[4$, $5]$. The benefits of neutral helium as a probe particle are exploited by helium atom scattering (HAS), a non-destructive surface structural tool whereby a monochromated beam of helium atoms is scattered from a sample. The resulting diffraction patterns reveal information about surface structure [6] and dynamic surface processes [7]. The low helium atom-surface interaction energy ensures unambiguous surface sensitivity, and even a degree of chemical sensitivity through further analysis of inelastic scattering events [1]. A Scanning Helium Microscope (SHeM) is then a spatially resolved form of HAS, creating detailed maps of surfaces while retaining the unique advantages of neutral helium as a probe particle. As such, the SHeM provides the opportunity for an imaging technique capable of examining the surface of delicate samples non-destructively, as well as offering a probe with a wavelength of the order of atomic dimensions [1].

Several technological issues have formed the major roadblock in the development of a practical neutral helium microscope, among them the complexity, cost and lack of reliability of atom beam optics. The inert and neutral nature of helium atoms makes focusing difficult, although reflective
[2], diffractive [3], and refractive [8] techniques have been demonstrated. Here we report a simple scanning helium microscope (SHeM) utilizing a high intensity supersonic freejet beam source and pinhole 'optics' in which the helium beam is simply collimated, rather than focused. The pinhole geometry overcomes the need for complex optical elements, however, the resolution is then limited by the diameter of the pinhole with any improvement in resolution causing a direct decrease in signal. Despite these limitations, the reported instrument successfully demonstrated the feasibility of a pinhole SHeM and has allowed for an initial investigation of the contrast mechanisms available to the technique.

\section{Experimental Setup}

\subsection{Design of a Pinhole SHeM}

The prototype SHeM presented here, shown schematically in Figure 1, was designed to be a proof-of-concept for the technique, as well as a means to further investigate the issue of helium contrast mechanisms. Having the beam strike the sample at 45 degrees and placing the detector aperture at a similar angle allows the SHeM to more readily differentiate between specular and diffuse reflections, a major source of contrast on a cleaned sample in HAS. The limitations of such a design are the large beam path length from source to sample to detector, and the working distance between pinhole and sample. Geometries in which the beam strikes normal to the surface are currently able to produce greater signal levels, but potentially limit the available contrast mechanisms [9]. Most importantly, the current geometry also affords the future potential to replacing the pinhole with a Fresnel zone plate to drastically improve the amount of signal. 
The source for the instrument consists of a supersonic freejet beam to provide an intense, well-collimated and nearly mono-energetic supply of neutral helium atoms. The source operates in an intermediate pressure regime [10] and consists of a nozzle (10 $\mu \mathrm{m}$ aperture) based on the design of Buckland et al [11] and a Beam Dynamics skimmer $(100 \mu \mathrm{m}$ aperture) that is used to sample the centerline atoms (producing the collimated beam) and separate the source chamber from the remainder of the apparatus. The nozzle can be moved with respect to the skimmer with an $x-y-z$ manipulator (UHV Designs) and the pressure behind the nozzle, known as the stagnation pressure, can be controlled with a resolution of 1 bar. The source chamber is evacuated by a Shimadzu TMU2203 2000 litre/second turbomolecular pump backed by an Edwards E2M80 rotary vane pump. The source is capable of producing a centerline intensity of $(1.4 \pm 0.1) \times 10^{20}$ atoms/second/steradian at 200 bar stagnation pressure, calculated from the source chamber exhaust gas flow rate [11]. Assuming an effective source size of $200 \mu \mathrm{m}$ [12], this corresponds to a source brightness of $(1.1 \pm 0.1) \times 10^{23}$ atoms $\mathrm{cm}^{-2} \mathrm{~s}^{-1} \mathrm{sr}^{-1}$. For comparison, the thermionic emission source in a typical electron microscope has brightness around $5 \times 10^{4} \mathrm{~A} \mathrm{~cm}^{-2} \mathrm{sr}^{-1}$ [2], or $3 \times 10^{23}$ electrons $\mathrm{cm}^{-2} \mathrm{~s}^{-1} \mathrm{sr}^{-1}$. Thus the helium source used in the SHeM has brightness comparable with the source used in an SEM or TEM. When the beam is in operation, the source chamber pressure typically rises from $10^{-8}$ mbar to $10^{-3}$ mbar.

A short, differentially pumped chamber separates the source chamber from the sample chamber, reducing the background helium gas load around the sample. The beam then passes through the limiting aperture, a $5 \mu \mathrm{m}$ diameter pinhole situated approximately $150 \mathrm{~mm}$ downstream from the nozzle, into the sample chamber. The pinhole itself is created by focused ion beam (FIB) milling through a silicon nitride membrane ( 250 by 250 micron window of $200 \mathrm{~nm}$ thickness, Ted Pella p.n. 21525) as shown in Figure 2. The beam is incident on the sample at 45 degrees, with a $3 \mathrm{~mm}$ working

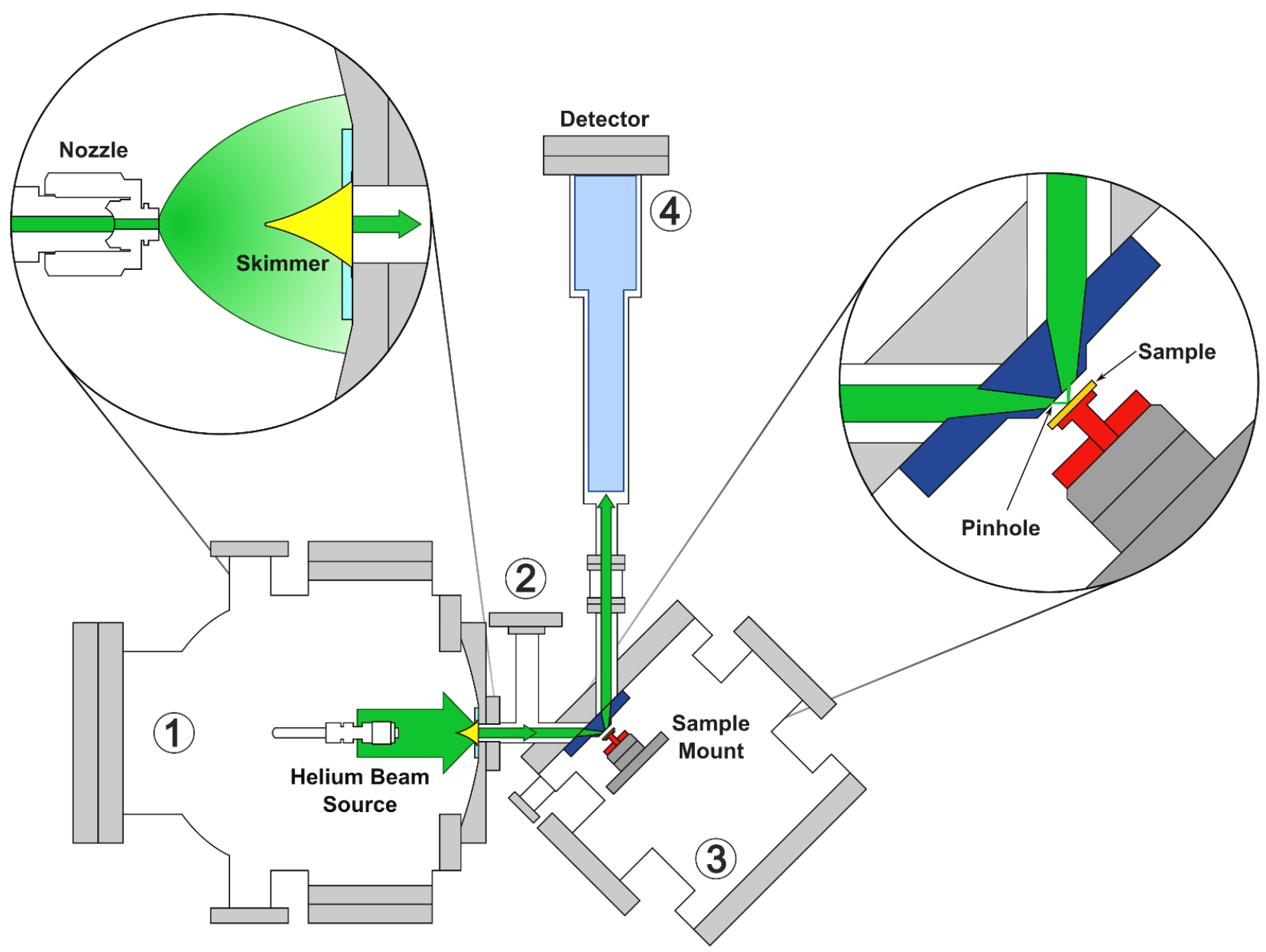

Figure 1: Schematic diagram of the prototype SHeM. The helium beam source consists of a free-jet expansion created with a 10 micron aperture before the centreline of the expansion is selected out with a 100 micron skimmer in the source chamber (1). The beam passes through a differential pumping stage (2) to the pinhole optics of the instrument, a 5 micron FIB milled pinhole in an Au-coated silicon nitride membrane. The result is a thin beam of helium striking the sample surface in the sample chamber (3), with the scattered helium entering the detector chamber (4) where it stagnates to form a stable pressure. By rastering the sample back and forth under the beam an image of the surface may be constructed. 
distance, after which it may scatter into the detector aperture ( $1 \mathrm{~mm}$ diameter also at 45 degrees to the sample). The sample chamber is pumped by a $520 \mathrm{~L} \mathrm{~s}^{-1}$ Pfeiffer TMU 521 turbomolecular pump, backed by a ??? rotary vane pump. Figure 3 shows a schematic diagram of the sample chamber scattering geometry. The sample is mounted to a stack of two Attocube ECS3030 piezo slip-stick drives, giving $\mathrm{x}$ and $\mathrm{y}$ positioning. The working distance is adjusted by means of a simple jackscrew.

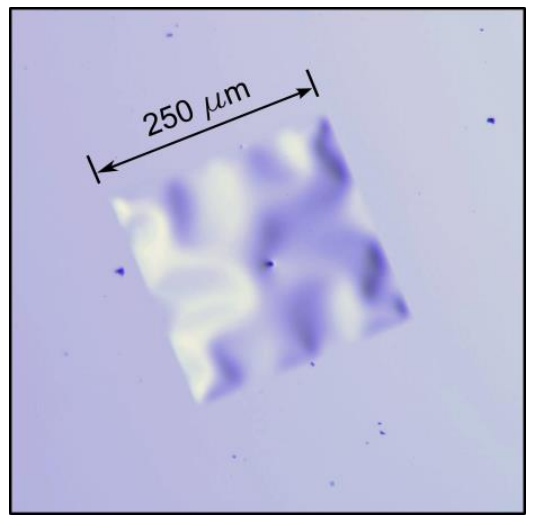

Figure 2: Optical micrograph of the pinhole which forms the optics for the SHeM.

The detector is of a stagnation design, and consists of a Hiden Analytical 3F-PIC residual gas analyzer (RGA), mounted within a close-fitting sheath. The sheath is separated from the sample chamber by a gate valve, allowing the detector to remain at UHV pressures whilst samples are interchanged. The RGA is set to a fixed mass/charge ratio of 4 , with the analog count rates output signal from the RF head conditioned with a Schmitt trigger and read via an Agilent $53131 \mathrm{~A}$ pulse counter. The detector sheath is pumped via a low, fixed conductance port of approximately $2 \mathrm{~L} \mathrm{~s}^{-1}$. In the free molecular flow regime, the rate of pressure change in a volume $V$ pumped at rate $S$ when gas load $Q$ is introduced is:

$$
-\mathrm{V}\left(\frac{\mathrm{dP}(\mathrm{t})}{\mathrm{dt}}\right)=\mathrm{S} \cdot \mathrm{P}(\mathrm{t})-\mathrm{Q}
$$

where $P(t)$ is pressure as a function of time. At equilibrium $(d P(t) / d t=0)$, the expression becomes:

$$
\mathrm{P}_{\mathrm{eq}}=\frac{\mathrm{Q}}{\mathrm{S}}
$$

For a fixed beam flux, $Q$ will then be the number of helium atoms entering the detector aperture per unit time. $Q$ is the sum of contributions from the different scattering processes, and hence will be made up of a diffuse, a specular and a background term. By scanning the sample stage in a raster pattern, the equilibrium pressure $\mathrm{P}_{\mathrm{eq}}$ will follow linearly $\mathrm{Q}$. Therefore by setting the RGA to measure the partial pressure of helium in the detector sheath, $\mathrm{Q}$ can be measured as a function of sample $x-y$ position. As the detector is of a stagnation design, there is a characteristic signal rise time with rate constant $S / V$. Thus the scan routine includes a wait time between pixels, taking into account this rise time and therefore allowing for an accurate measurement of $\mathrm{Q}$.

\subsection{Characterisation of the Instrument}

The SHeM was characterized over a range of stagnation pressures (10-200 bar), detector emission currents (20-300 $\mu \mathrm{A})$ and nozzle-to-skimmer alignments. While the primary source for the neutral atoms striking the sample surfaces is the direct supersonic free jet beam sampled by the skimmer, it was found that there was a secondary effusive beam at play in the instrument. During beam operation, the background helium pressure in the differentially pumped stage increases such that the mean free path of the helium atoms within the chamber become comparable with the dimensions of the pinhole. As a result, helium gas effuses through the pinhole in a broad cosine distribution. This effusive beam (produced similarly to the beam from a Knudsen cell) effectively broadens the helium beam, degrading instrument resolution. By optimising the nozzle-to-skimmer separation it was possible to reduce the pressure in the differentially pumped stage so as to have the free-jet beam dominate the effusive beam, at the cost of the total signal detected, as shown below in Figure 3.

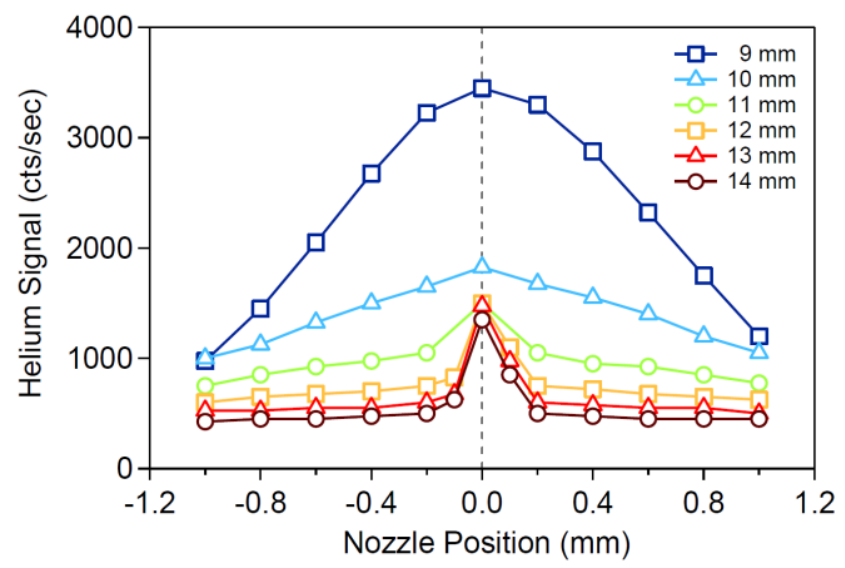

Figure 3: The detected helium signal as a function of varying nozzle-to-skimmer separations. At small separations, the pressure in the differential chamber increases to the point where an effusive beam is produced in the sample chamber and dominates the supersonic beam. However, there is an optimum nozzle-to-skimmer distance where the free-jet beam dominates the effusive beam, as can be seen by the emergence of a strong dependency on the nozzle position relative to the skimmer. The nozzle-to-skimmer separations have a zero offset of up to $1 \mathrm{~mm}$ due to the difficulty of aligning the nozzle with the fragile skimmer.

Line scans were taken over the sharp edge of a TEM grid to ascertain the SHeM's resolution. Figure 4 shows the average of four line scans taken over the step edge. Deconvolution of the line scan with the step edge indicates an instrument resolution of $5 \pm 1 \mu \mathrm{m}$. 
The SHeM's performance was modelled using free-molecular gas flow dynamics $[2,13,14]$. The gas flow through each aperture was modelled with both a free-jet and effusive component as a function of chamber pressure.

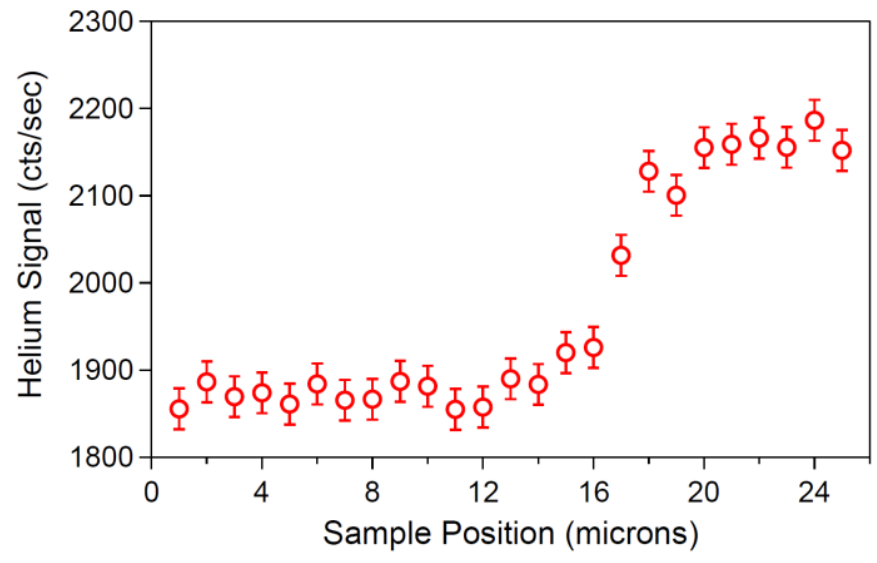

Figure 4: Line scan over a step edge (TEM grid), showing the convolution of the beam's line spread function with the edge.

The scattering from the sample was modelled conservatively as purely diffuse, with no specular component. Table 1 shows a comparison of the experimental and modelled performance of the SHeM.

\section{Imaging Results and Discussion}

The SHeM was used to investigate some initial sample systems with an aim toward understanding the contrast mechanisms available to neutral helium atom microscopy. The SHeM was first calibrated by imaging a TEM grid (see Figure 5a). Following this TEM grid study, a number of systems were investigated, such as tin spheres on a carbon substrate (Figure 5c) and flat-polished polymer bonded explosives (Figure 5e).

All of the samples imaged demonstrated clear evidence for topological contrast as a function of the angle of sample surface normal at each pixel relative to the entrance angle of
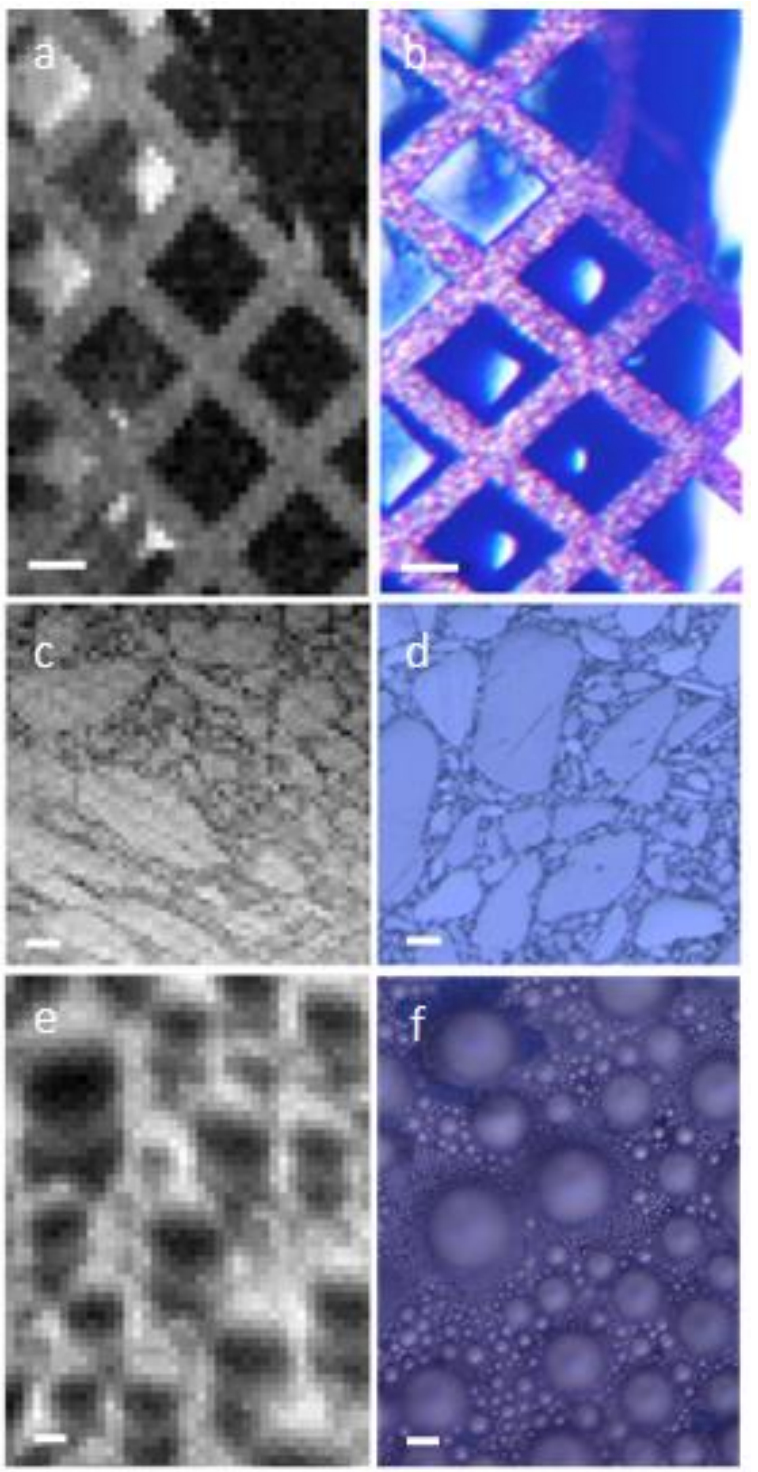

Figure 5: SHeM (a) and matched reflection optical microscope image (b) of a broken copper TEM grid with 20 $\mu \mathrm{m}$ bar width and $80 \mu \mathrm{m}$ periodicity, mounted on carbon tape. SHeM (c) and optical microscope (d) image of polymer bonded explosives, polished flat to within $50 \mathrm{~nm}$. SHeM (e) and optical microscope (f) image of tin spheres on carbon. All scales bars are $30 \mu \mathrm{m}$.

Table 1: Performance characteristics of the SHeM at 200 bar nozzle stagnation pressure.

\begin{tabular}{|l|l|l|}
\hline Parameter & Model & Experimental \\
\hline Beam centreline intensity & $1.46 \times 10^{20} \mathrm{He} \mathrm{s}^{-1} \mathrm{sr}^{-1}$ & $(1.4 \pm 0.1) \times 10^{20} \mathrm{He} \mathrm{s}^{-1} \mathrm{sr}^{-1}$ \\
\hline Scan resolution & $5.0 \mu \mathrm{m}$ & $5 \pm 1 \mu \mathrm{m}$ \\
\hline Detected count rate & 2607 counts s$^{-1}$ & $1500-4000 \mathrm{counts} \mathrm{s}^{-1} \dagger$ \\
\hline Detected signal-to-background & 1.63 & $1.15 \pm 0.10$ \\
\hline Detected signal-to-noise & 40.21 & $28-42 \dagger$ \\
\hline
\end{tabular}

† Count rates and signal-to-noise ratio dependent on sample. 
the detector aperture. Surfaces and edges oriented towards the detector aperture typically appear to be the 'brightest' in the produced micrographs. Therefore the main source of this topological image contrast is likely diffuse scattering, arising due to surface roughness. A subset of this contrast mechanism is shadowing, whereby a surface asperity completely occludes the detector aperture. Strong shadowing contrast was also present in all images, particularly on the tin spheres sample. The maximum contrast in the helium signal level from white to black in all of the samples was found to be between 15 and $25 \%$.

Despite the 45 degree specular scattering geometry, little evidence for contrast mechanisms other than topological was found. It is well known from HAS studies that the amount of helium scattered inelastically (critical to the chemical sensitivity of the technique) is 2-3 orders of magnitude smaller than that scattered elastically [15]. As such, count rate differences between materials may have been beneath the noise floor of the current instrument - a problem which would be exacerbated by the secondary effusive beam providing a low intensity source of helium atoms to a wider area of the sample surface. Alternatively, there is the possibility that due to the unambiguous surface sensitivity of the technique, even a single monolayer of water or hydrocarbons could sufficiently degrade the sample's specular peak such that diffuse scattering would dominate. As such, a future instrument may require a means of in situ sample cleaning, such as a cluster ion source [16], so as to take advantage of the other contrast mechanisms available to helium atom scattering.

\section{Conclusions:}

In this paper we have presented the design for a simple pinhole scanning helium microscope (SHeM), which produces images by detecting neutral helium atoms scattered from a sample. The SHeM performance was modelled using free-molecular gas dynamics, with the experimental results found to be in good agreement with the model. The instrument resolution is set by the diameter of the final collimation aperture (the pinhole) and so currently offers only a modest resolution of about $5 \mu \mathrm{m}$. However the modular nature of the system allows for resolution improvements by decreasing the pinhole diameter, or by the replacement of the pinhole with a free-standing Fresnel zone plate. Further work is currently underway on a refined version of the instrument.

\section{Acknowledgements:}

This research was supported under the Australian Research Council's Discovery Projects (project number DP08831308) and Linkage International Fellowships (project number
LX0990077) funding schemes. Postgraduate research scholarships (MB, AF) from the University of Newcastle and travel funding from the Australian Nanotechnology Network are gratefully acknowledged. We thank the Cavendish workshop for technical support and assistance. We also thank Chris Wade and Joel Martens for additional support on this project.

\section{References:}

[1] MacLaren D A, Holst B, Riley D J and Allison W 2003 Focusing elements and design considerations for a scanning helium microscope (SHeM) Surf. Rev. Lett. 10 249-55

[2] Maclaren D A 2002 Development of a single crystal mirror for scanning helium microscopy $P h D$ Thesis University of Cambridge

[3] Koch M, Rehbein S, Schmahl G, Reisinger T, Bracco G, Ernst W and Holst B 2008 Imaging with neutral atoms-a new matterwave microscope J. Microsc. 229 1-5

[4] O’Donnell K M 2009 Field ionization detection for atom microscopy PhD Thesis University of Newcastle, New South Wales

[5] Doak R B, Grisenti R E, Rehbein S, Schmahl G, Toennies J P and Wöll C 1999 Towards realization of an atomic de Broglie microscope: helium atom focusing using Fresnel zone plates Phys. Rev. Lett. 83 4229-32

[6] Traeger F 2006 Helium atom scattering from oxide surfaces ChemPhysChem. 7 1006-13

[7] Jardine A, Ellis J and Allison W 2002 Quasi-elastic helium-atom scattering from surfaces: experiment and interpretation J. Phys.: Condens. Matter. 14 6173-91

[8] Jardine A P, Fouquet P, Ellis J, and Allison W 2001 Hexapole magnet system for thermal energy $3 \mathrm{He}$ atom manipulation Rev. Sci. Instruments 72 3834-3841

[9] Witham P and Sánchez E 2011 A simple approach to neutral atom microscopy Rev. Sci. Instrum. 82103705

[10] Barr M, O’Donnell K M, Fahy F, Allison W and Dastoor P C 2012 A desktop supersonic free-jet beam source for a scanning helium microscope (SHeM) Meas. Sci. Technol. 23105901

[11] Buckland J R, Folkerts R L, Balsod R B and Allison W 1997 A simple nozzle design for high speed-ratio molecular beams Meas. Sci. Technol. 8 933-5

[12] Holst B 1997 Atom optics and surface growth studies using helium atom scattering PhD Thesis University of Cambridge

[13] Beijerinck H C W and Verster N F 1981 Absolute intensities and perpendicular temperatures of supersonic beams of polyatomic gases Physica C 111 327-52

[14] Chambers A 2004 Modern Vacuum Systems CRC Press Florida

[15]Doak R B 1992 Single Phonon Inelastic Helium Scattering, in Atomic and Molecular Beam Methods, Vol 2 (ed: Scoles G) Oxford University Press New York

[16] Ninomiya S, Nakata Y, Ichiki K, Seki T, Aoki T and Matsuo J 2007 Measurements of secondary ions emitted from organic compounds bombarded with large gas cluster ions Physics Research Section B 256 492-496 